\title{
Super-accelerating bouncing cosmology in asymptotically free non-local gravity
}

\author{
Gianluca Calcagni $^{1, \mathrm{a}}$, Leonardo Modesto ${ }^{2, \mathrm{~b}}$, Piero Nicolini $^{3, \mathrm{c}}$ \\ ${ }^{1}$ Instituto de Estructura de la Materia, CSIC, Serrano 121, 28006 Madrid, Spain \\ 2 Department of Physics and Center for Field Theory and Particle Physics, Fudan University, Shanghai 200433, China \\ ${ }^{3}$ Frankfurt Institute for Advanced Studies (FIAS) and Institut für Theoretische Physik, Johann Wolfgang Goethe-Universität, \\ 60438 Frankfurt am Main, Germany
}

Received: 12 April 2014 / Accepted: 25 July 2014 / Published online: 21 August 2014

(C) The Author(s) 2014. This article is published with open access at Springerlink.com

\begin{abstract}
Recently, evidence has been collected that a class of gravitational theories with certain non-local operators is renormalizable. We consider one such model which, at the linear perturbative level, reproduces the effective non-local action for the light modes of bosonic closed string-field theory. Using the property of asymptotic freedom in the ultraviolet and fixing the classical behavior of the scale factor at late times, an algorithm is proposed to find general homogeneous cosmological solutions valid both at early and late times. Imposing a power-law classical limit, these solutions (including anisotropic ones) display a bounce, instead of a big-bang singularity, and super-accelerate near the bounce even in the absence of an inflaton or phantom field.
\end{abstract}

\section{Introduction}

Asymptotic freedom is an attribute of field theories such that interactions are negligible in the ultraviolet (UV), where the theory possesses a trivial fixed point. This property has been used, directly or implicitly, to construct field theories of gravity where the Laplace-Beltrami operator $\square$ is replaced by one or more non-local operators $f(\square)$ in the kinetic terms of the action. On a Minkowski background, if the Fourier transform $\tilde{f}\left(k^{2}\right) \rightarrow \infty$ for large momenta $k$, these terms dominate in the UV over the interactions, which can be ignored. Asymptotic freedom then, if realized, ensures that the correct UV behavior of the theory be encoded in the free propagator. A class of these models is of particular relevance inasmuch as the kinetic operator is of the exponential form $\square \mathrm{e}^{\square}$ or $\mathrm{e}^{\square}$ and is inspired, respectively, by open string-field theory

\footnotetext{
a e-mail: calcagni@iem.cfmac.csic.es

b e-mail:1modesto@fudan.edu.cn

c e-mail: nicolini@fias.uni-frankfurt.de
}

(OSFT; see [1-4] for reviews) and the $p$-adic string [5-10]. In the simplest classical cosmological applications, gravity is local and the only non-local content is a scalar field [11-35], sometimes identified with the tachyon of bosonic OSFT or of super-symmetric OSFT on an unstable brane.

Non-local gravity sectors and their cosmology have been proposed in [36-57], following various criteria including avoidance of ghosts, improved renormalizability, and the possibility to construct non-perturbative solutions. The gathered results (also in theories without non-local operators at the tree level [58]) point towards a resolution of gravitational singularities thanks to asymptotic freedom. These approaches do not stem from closed string-field theory, i.e., the SFT sector containing the perturbative graviton mode [59-73]. Yet, many of them are inspired by it in the sense that the kinetic functions are exponential (or somewhat more general) operators as in effective closed SFT (see also [74-78] for other types of non-locality). In this context, one bypasses the technical difficulties in getting effective non-local actions directly from SFT $[62,73]$ and concentrates on phenomenological but more manageable models.

Cosmological dynamical solutions were obtained either directly, by solving the non-local equations of motion, or indirectly, by solving an Ansatz for the Ricci curvature (or via the diffusion equation [44]) respecting the equations of motion. Here we pursue a different but no less economic alternative, using asymptotic freedom as a key ingredient.

The strategy is the following. (i) First, in Sect. 2 we define a model of non-local gravity inspired by closed SFT and falling into the class of actions considered in $[45,47]$. (ii) In the UV, all interactions can be ignored and, thus, it is sufficient to find cosmological backgrounds compatible with the Green equation for the propagator. The scale factors $a(t)$ representing such backgrounds do not collapse into a big bang 
but, rather, display a bounce. At large scales, they reduce to known profiles of ordinary cosmology, the details of which depend on the choice of matter content. In particular, in Sect. 3 we will find, with two different methods, backgrounds following a power law at late times. These profiles are approximate solutions of the full equations of motion valid both at very early times (when, roughly, the cosmological horizon scale is near the UV asymptotically free fixed point) and at late times. All such solutions have a bounce and accelerate near it without invoking inflaton-like matter content. ${ }^{1}$ (iii) Next, we write down an effective Friedmann equation of the form

$H^{2}=\frac{\kappa^{2}}{3} \rho_{\mathrm{eff}}:=\frac{\kappa^{2}}{3} \rho\left[1-\left(\frac{\rho}{\rho_{*}}\right)^{\beta}\right]$,

where $H:=\dot{a} / a=\partial_{t} a / a$ is the Hubble parameter, $\kappa^{2}=$ $8 \pi G, G$ is Newton's constant, $\rho$ is the energy density of the universe, $\rho_{*}$ is the critical energy density at which the bounce occurs, and $\beta>0$ is a real parameter. The exponent $\beta$ is determined by plugging the profile $a(t)$ found under the provision of asymptotic freedom into Eq. (1) for a given energy density profile $\rho(a)$. Since this fitting is generally rather good, we can conclude that the class of asymptotic solutions found in step (ii) is reasonably valid also at intermediate times, and that the bouncing-accelerating scenario of the theory is well described by the effective Friedmann equation (1).

\section{The model}

\subsection{Effective action from closed string-field theory}

We start by recalling the derivation of the effective SFT Lagrangian for the closed string through a mass-level truncation scheme. In the non-polynomial bosonic closed SFT, the action has the following compact form:

$S_{\mathrm{SFT}}=\int \frac{1}{\alpha^{\prime}} \Phi * Q b_{0}^{-} \Phi+g \sum_{N=3}^{+\infty} \frac{\left(g \alpha^{\prime}\right)^{N-3}}{2^{N-3} N !} \Phi *\left[\Phi^{N-1}\right]$,

where $\Phi$ is the closed-string field, $Q$ is the BRST operator, $b_{0}^{-}=\left(b_{0}-\bar{b}_{0}\right) / 2$ is a combination of antighost zero modes, $\alpha^{\prime}$ is the Regge slope and $g$ is the string-field coupling constant. The integral $\int \Phi_{1} * \Phi_{2}$ represents the string-field scalar product, while the symbol $\left[\Phi^{N-1}\right]=\left[\Phi_{1} \ldots \Phi_{N-1}\right]$ denotes the string field obtained combining $N-1$ fields $\Phi_{1}, \ldots, \Phi_{N-1}$ using the $N$-string vertex function.

\footnotetext{
${ }^{1}$ See [79-81] for inflation-without-inflaton scenarios descending from other mechanisms.
}

The idea that light states dominate physical processes justifies the following truncation of the string field in terms of oscillators and particle fields:

$$
\begin{gathered}
\Phi=c_{0}^{-}\left[\phi+A_{\mu \nu} \alpha_{-1}^{\mu} \bar{\alpha}_{-1}^{\nu}+\frac{\left(\alpha_{+}+\alpha_{-}\right) b_{-1} \bar{c}_{-1}}{\sqrt{2}}+\frac{\left(\alpha_{+}-\alpha_{-}\right) c_{-1} \bar{b}_{-1}}{\sqrt{2}}\right. \\
\left.+\mathrm{i} c_{0}^{+}\left(j_{1 \mu} \alpha_{-1}^{\mu} \bar{b}_{-1}+j_{2 \mu} b_{-1} \bar{\alpha}_{-1}^{\mu}\right)\right]|0\rangle
\end{gathered}
$$

where $A_{(\mu \nu)}:=\left(A_{\mu \nu}+A_{\nu \mu}\right) / 2$ is the graviton field, $A_{[\mu \nu]}:=\left(A_{\mu \nu}-A_{\nu \mu}\right) / 2$ is an antisymmetric rank-2 tensor field, $\alpha_{ \pm}, j_{1 \mu}$ and $j_{2 \mu}$ are auxiliary fields, and $b$ and $c$ are first-quantized ghost oscillators. Greek indices run over spacetime directions and are lowered via the Minkowski met$\operatorname{ric} \eta_{\mu \nu}=\operatorname{diag}(+,-, \ldots,-)$. The state $|0\rangle=\bar{c}_{1}|\bar{\Omega}\rangle \otimes c_{1}|\Omega\rangle$ is the first-quantized string vacuum, with $|\bar{\Omega}\rangle$ and $|\Omega\rangle$ the left and right $S L(2, \mathbb{R})$-invariant vacua. Further details can be found, e.g., in [1].

The truncation (3) allows one to derive the cubic effective Lagrangian $\mathcal{L}=\mathcal{L}_{\text {free }}+\mathcal{L}_{\text {int }}$. Working in the SiegelFeynman gauge $b_{0}^{+} \Phi=0$ (which sets $j_{1 \mu}=j_{2 \mu}=0$ ), the kinetic and mass terms read

$$
\begin{aligned}
\mathcal{L}_{\text {free }}= & \frac{1}{2} \partial_{\lambda} A_{\mu \nu} \partial^{\lambda} A^{\mu \nu}+\frac{1}{2} \partial_{\lambda} \phi \partial^{\lambda} \phi+\frac{2}{\alpha^{\prime}} \phi^{2} \\
& +\frac{1}{2} \partial_{\lambda} \alpha_{+} \partial^{\lambda} \alpha_{+}-\frac{1}{2} \partial_{\lambda} \alpha_{-} \partial^{\lambda} \alpha_{-},
\end{aligned}
$$

while $\mathcal{L}_{\text {int }}=\mathcal{L}_{\text {int }}\left(\partial, \tilde{\phi}, \tilde{A}, \tilde{\alpha}_{ \pm}\right)$has a number of interaction terms with derivatives. For a given field $\varphi(x)$ in the kinetic term, we have the corresponding "dressed" field

$\tilde{\varphi}(x)=\mathrm{e}^{-\square /\left(2 \Lambda^{2}\right)} \varphi(x), \quad \square=\partial_{\mu} \partial^{\mu}$

in the interaction part, where $1 / \Lambda^{2}=\alpha^{\prime} \ln (3 \sqrt{3} / 4) \approx$ $0.2616 \alpha^{\prime}$. Operators of this form are a fully non-perturbative effect of the string interactions. The conformal field theory describing such interactions is based upon special Fock states which obey a universal diffusion equation; such a structure is inherited by the effective spacetime action, as a residual manifestation of OSFT gauge invariance [82]. Non-local operators are, in general, associated with extended objects (rather than pointwise particles). Specifically, the exponential operators of the type considered in this paper are an imprint of the finite size of the string [83].

The above Lagrangian $\mathcal{L}$ can be recast in an equivalent form by shifting the smearing functions from the interaction term to the kinetic and mass ones by the field redefinition $\varphi(x) \rightarrow \mathrm{e}^{\square /\left(2 \Lambda^{2}\right)} \varphi(x)$. Ignoring the auxiliary fields, the free part of the Lagrangian reads

$\mathcal{L}_{\text {free }}=-\frac{1}{2} A_{\mu \nu} \square \mathrm{e}^{\square / \Lambda^{2}} A^{\mu \nu}-\frac{1}{2} \phi\left(\square-\frac{2}{\alpha^{\prime}}\right) \mathrm{e}^{\square / \Lambda^{2}} \phi$, 
which leads to the following stringy modifications of the Laplace-Beltrami operator in the zero-level truncation scheme:

$\square \rightarrow \square \mathrm{e}^{\square / \Lambda^{2}}$.

The associated propagator generally leads to a ghost-free spectrum, the intuitive reason being that entire functions $f(\square)$ do not introduce extra poles $[14,25,42,84]$. Quantum field theories with exponential propagators have been argued long since to be super-renormalizable [85]. Operators of the form (7) also appear in the context of non-commutative geometries, where a minimal length is effectively induced [86-89].

\subsection{Non-local gravity model}

From the tree-level effective action for the graviton and matter, we can argue about the form of its non-linear covariant extension. On the ground of a recently introduced candidate model of super-renormalizable gravity $[45,47,48,50,53,54]$ based on earlier results $[36,37]$ (see also [90] for considerations in a local higher-derivative theory), we propose the action $^{2}$

$$
\begin{gathered}
S=\frac{1}{2 \kappa_{D}^{2}} \int \mathrm{d}^{D} x \sqrt{|g|}\left[R-G_{\mu \nu} \gamma(\square) R^{\mu \nu}\right]+S_{\text {matter }}, \\
S_{\text {matter }}=\int \mathrm{d}^{D} x \sqrt{|g|}\left[\frac{1}{2} \nabla_{\mu} \phi V^{-1}(\square) \nabla^{\mu} \phi\right. \\
\left.\quad+\frac{1}{2 n !} \mathrm{e}^{c \phi} F_{[n]} V^{-1}(\square) F_{[n]}\right],
\end{gathered}
$$

where $G_{\mu \nu}$ and $R_{\mu \nu}$ are, respectively, the Einstein tensor and the Ricci tensor associated with the $D$-dimensional target spacetime metric $g_{\mu \nu}$, and, adopting the terminology used in string theory, $\phi$ is the dilaton field coming from the trace of the field $A_{\mu \nu}, n=p+2$, and $F_{[n]}$ are the $p$-field strengths corresponding to the gauge potentials. The value of the parameter $c$ controls the interaction of the scalar field $\phi$ with the field strength $F_{[n]}$. The key ingredients of the above action are the operators

$\gamma(\square):=\frac{V^{-1}(\square)-1}{\square}, \quad V(\square):=\mathrm{e}^{-\square / \Lambda^{2}}$,

where $\Lambda$ (proportional to $\alpha^{\prime-1 / 2}$ in SFT) is the invariant energy scale above which quantum-gravity effects become non-negligible. This form of the kinetic terms correctly reproduces the non-local operator (7) (and its inverse, the propagator $V(\square) / \square$ ) when linearizing the fields [36,37]. It also leads to the improved renormalizability of the model.

\footnotetext{
2 This action differs from the theory of [44], which is of scalar-tensor type. There, the curvature invariants appear only in the exponential non-local operator in order to allow for non-perturbative solutions via a method based on the diffusion equation.
}

However, in a spacetime of even dimension the effective action is not generally finite but only super-renormalizable because one-loop diagrams are still superficially divergent $[36,37,47,48,50,53,54,90,91]$. Things go differently in a spacetime of odd dimension, because at the one-loop level there are no local operators which can serve as counterterms for pure gravity and the theory results to be finite $[92,93]$. When, in the case of super-symmetry, matter is added to fill up the super-gravity multiplet, the theory remains finite [94].

From the point of view of string-field theory, the action (8) only contains massless fields, but in general the whole tower of string massive particle modes should be taken into account. However, for the cosmology-related purposes of the present paper the massless sector is more than enough, since gravity is included in it and, on the other hand, cosmological matter can be modeled by a perfect fluid as a first approximation, even if at the fundamental level it is constituted by fields with non-local dynamics. In this regard, notice that the form of the kinetic operators in (8) is expected to hold also for massive modes [93] (consult that reference for a longer discussion). One simple example of this is given by the string tachyon, which is a massive field although with negative squared mass; as one can see from Eq. (6), its kinetic operator is exactly of the same form as for the massless fields. Another motivation to drop massive states from the discussion is that a perturbative truncation of the non-local operators would correspond to a small Regge slope. By keeping these operators intact we imply no special requirement on the size of squared momenta $k^{2}$ with respect to $\alpha^{\prime}$. In particular, one is entitled to explore configurations such that $\alpha^{\prime} k^{2} \gg 1$. This is the region in parameter space where all the masses of the tower, which are multiples of the fundamental mass $1 / \sqrt{\alpha^{\prime}}$, can be ignored with respect to kinetic terms $\propto k^{2} \gamma\left(-k^{2}\right)$. Therefore, a truncation of the massive tower is in principle compatible with keeping the non-local operators untruncated.

In this paper, we study asymptotic profiles which are approximate cosmological solutions of the gravitational system (8a). The classical action (8a) is a "non-polynomial" or "semi-polynomial" extension of quadratic Stelle theory $[90,95]$. All the non-polynomiality is incorporated in the form factor $\gamma(\square)$. The entire function $V$ has no poles in the whole complex plane, which preserves unitarity, and it has at least logarithmic behavior in the UV regime to give super-renormalizability at the quantum level.

Here we only consider corrections to the classical solutions coming from the bare two-point function of the graviton field. The reason is that this class of theories is asymptotically free and the leading asymptotic behavior of the dressed propagator is dominated by its bare part. In fact, according to power counting arguments [37,47], the self-energy insertions, which are constant or at most logarithmic, do not contribute to it. 


\section{Cosmology}

\subsection{General solution in asymptotically free gravity}

To find general homogeneous and isotropic cosmological solutions in the UV, we adapt a procedure used in ordinary perturbative quantum gravity [96-98]. Later on, we shall comment on the main differences of our setting with respect to earlier applications. The same method can be applied also in the case of gravitational collapse [99]. We fix the number of dimensions to $D=4$. Consider a homogeneous and isotropic Friedmann-Robertson-Walker (FRW) metric $g_{\mu \nu}$ with zero curvature; the anisotropic case is straightforward and will be discussed later. We split the metric into a flat Minkowski background plus a homogeneous tensor $h_{\mu \nu}$,

$$
\begin{aligned}
g_{\mu \nu} & =\eta_{\mu \nu}+\kappa h_{\mu \nu}, \mathrm{d} s^{2}=g_{\mu \nu} \mathrm{d} x^{\mu} \mathrm{d} x^{\nu} \\
& =Z \mathrm{~d} t^{2}-a(t)^{2} \delta_{i j} \mathrm{~d} x^{i} \mathrm{~d} x^{j}
\end{aligned}
$$

where $i=1,2,3$. By definition, $h$ is small around a certain time $t_{\mathrm{i}}$, where $g_{\mu \nu}\left(t_{\mathrm{i}}\right)=\eta_{\mu \nu}$, but only then. In fact, the Ansatz (10) is not to be understood in the sense of cosmological perturbation theory, where $\kappa h \ll 1$ is a perturbation small everywhere and at any time. Equation (10) is simply a splitting of the FRW metric, not the perturbation of Minkowski background with a generic fluctuation. Therefore, we stress that the present method has nothing to do with cosmological perturbation theory. ${ }^{3}$

Thus, the scale factor $a$ and the fluctuation $h_{\mu \nu}$ are

$$
\begin{aligned}
& a^{2}(t)=1-\kappa h(t), \quad h\left(t=t_{\mathrm{i}}\right)=0, \\
& g_{\mu \nu}\left(t=t_{\mathrm{i}}\right)=\eta_{\mu \nu}, \\
& h_{\mu \nu}(t)=h(t) \operatorname{diag}\left(0, \delta_{i j}\right)=: h(t) \mathcal{I}_{\mu \nu} .
\end{aligned}
$$

The tensor $h_{\mu \nu}$ can be rewritten in harmonic gauge by the transformation

$h_{\mu \nu}(x) \rightarrow h_{\mu \nu}^{\prime}(x):=h_{\mu \nu}(x)+\partial_{\mu} \xi_{\nu}+\partial_{\nu} \xi_{\mu}$,

$\xi_{\mu}(t)=-\frac{3 \kappa}{2} \operatorname{diag}\left[\int_{t_{\mathrm{i}}}^{t} d t^{\prime} h\left(t^{\prime}\right), 0,0,0\right]$.

The fluctuation now reads

$h_{\mu \nu}^{\prime}(t)=h(t) \operatorname{diag}\left(-3, \delta_{i j}\right), \quad h_{\mu}^{\prime \mu}(t)=-6 h(t)$.

The standard gravitational field $\bar{h}_{\mu \nu}$ is then

$$
\begin{gathered}
\bar{h}_{\mu \nu}:=h_{\mu \nu}^{\prime}-\frac{1}{2} \eta_{\mu \nu} h_{\lambda}^{\prime \lambda}=h(t) \operatorname{diag}\left(0,-2 \delta_{i j}\right)=-2 h(t) \mathcal{I}_{\mu \nu}, \\
\partial^{\mu} \bar{h}_{\mu \nu}=0 .
\end{gathered}
$$

\footnotetext{
3 Throughout the paper, and unless stated otherwise, we will use the term "perturbation theory" exclusively in the sense of field theory, not of cosmology.
}

The Fourier transform of the above field is given by

$\tilde{\bar{h}}_{\mu \nu}(E, \mathbf{k})=-2 \tilde{h}(E)(2 \pi)^{3} \delta(\mathbf{k}) \mathcal{I}_{\mu \nu}$.

At this point, we exploit asymptotic freedom to avoid solving the full equations of motions of (8) on a flat FRW background, and to recognize the profile (11) as an actual asymptotic solution of our model. We express the classical propagator for the excitation $\bar{h}_{\mu v}$ via a dimensionless source $\varrho$, representing the 00 component of an effective "energymomentum" tensor $\mathcal{T}^{\mu \nu}$. Denote its Fourier transform with a tilde. The gauge-independent part of the graviton propagator $[47,100]$ for the theory $(8 \mathrm{a})$ is then

$\mathcal{O}^{-1}(k)=\frac{V\left(k^{2}\right)}{k^{2}}\left(P^{(2)}-\frac{P^{(0)}}{2}\right) \Longrightarrow$
$\bar{h}_{\mu \nu}(x)=\kappa \int \frac{\mathrm{d}^{4} k}{(2 \pi)^{4}} \mathcal{O}_{\mu \nu, \rho \sigma}^{-1}(k) \tilde{\mathcal{T}}^{\rho \sigma}(k) \mathrm{e}^{-\mathrm{i} k \cdot x}$,

where $P^{(0)}$ and $P^{(2)}$ are Van Nieuwenhuizen projectors in four dimensions [101]. The standard "classical" case is obtained for $V\left(k^{2}\right) \rightarrow 1$, but for the theory (8a) we find, using the graviton propagator after a Wick rotation,

$$
\begin{aligned}
h(t) & =-\frac{\kappa}{2} \int \frac{\mathrm{d}^{4} k}{(2 \pi)^{4}} \frac{1}{k^{2} V^{-1}\left(k^{2}\right)} \tilde{\varrho}(E, \mathbf{k}) \mathrm{e}^{-\mathrm{i} k \cdot x} \\
& =\int \frac{\mathrm{d} E}{2 \pi} \tilde{h}(E) \mathrm{e}^{\mathrm{i} E t} .
\end{aligned}
$$

Assuming that the kernel $\tilde{\varrho}$ does not depend on the cut-off $\Lambda$, the quantum-corrected profile $a(t)$ can be found from its "classical" limit $a_{\mathrm{cl}}(t)$. The procedure is the following: (i) fix an $a_{\mathrm{cl}}(t)$ and, via Eq. (11), a profile $h_{\mathrm{cl}}(t)$; (ii) set temporarily $V=1$ in Eq. (16) and obtain $\tilde{h}(E)$ and, from that, the cutoff-independent distribution $\tilde{\varrho}(E, \mathbf{k})$; (iii) plug $\tilde{\varrho}$ back into Eq. (16) and perform the four-momentum integral with the full $V\left(k^{2}\right) \neq 1$, to obtain $h(t)$; (iv) use (11) to get $a(t)$. The profile $a_{\mathrm{cl}}(t)$ is thus recovered perturbatively at times $t \gg t_{\mathrm{i}}$.

This procedure is similar to the one employed in [96-98], but with an important difference. There, in order to go beyond the classical theory, one introduces one-loop quantum corrections to the graviton propagator. In our case, however, we already have modifications at the classical level and, therefore, we use only the bare propagator. This can be justified by noting, as mentioned above, that one-loop corrections to the propagator in this class of non-local theories are UV subdominant with respect to the tree-level contribution. Thus, a general conclusion is that any asymptotically free theory of gravity with a two-point function of the form (15), with sufficiently strong damping factor $V$, will admit an asymptotic UV solution of the form (11) solely found at the tree level in perturbation theory. 


\subsection{General solution with power-law regime}

We start by considering a profile compatible, at late times, with a power law. We recall that we are not in vacuum and the matter source shapes the expansion of the universe. The classical profile is very simple, namely,

$a_{\mathrm{cl}}(t)=\left|\frac{t}{t_{\mathrm{i}}}\right|^{p}, \quad h_{\mathrm{cl}}(t)=\frac{1}{\kappa}\left[1-\left|\frac{t}{t_{\mathrm{i}}}\right|^{2 p}\right]$,

where $t_{\mathrm{i}}$ is the pivot time around which one centers the splitting of the metric, $t=0$ is the big-bang singularity time, and $p>0$ is a constant. Equation (17) is solution to the ordinary Einstein equations, recovered at low curvature also in our model when higher-order Riemann terms are negligible. $a_{\mathrm{cl}}(t)$ is an even function of time due to time-reversal symmetry in the standard classical Friedmann equations and, in turn, it will determine an even quantum-corrected profile $a(t)=a(|t|)$. This will also prevent a possible issue with the procedure (i) - (iv) detailed above. The propagator is integrated after Wick rotation, when the form factor $V$ makes it convergent. For the purpose of field-theory calculations, going to imaginary time poses no particular problem, provided the Osterwalder-Schrader conditions are satisfied [37, Section 5]. However, the solution $a(t)$ is found with the Wick-rotated propagator, and its analytic continuation back in Lorentzian time $t \rightarrow-$ it may no longer be sensible for the Lorentzian system. In the present case, however, the solution depends on $t^{2}=|t|^{2}$ ( $t$ is a real parameter) and it correctly reaches the classical power-law solution of the Lorentzian theory at late times. Therefore, the profile $a(t)$ is unaffected by the analytic continuation. In Sect. 3.3, we will recover the same solution with an independent method.

Given the condition (17), we can calculate the Fourier transform $\tilde{h}(E)$ defined in Eq. (14),

$\tilde{h}(E)=\frac{1}{\kappa E^{2} V^{-1}\left(E^{2}\right)}\left[\pi E^{2} \delta(E)+\frac{\sin (\pi p) \Gamma(2 p+1)}{t_{\mathrm{i}}^{2 p}|E|^{2 p-1}}\right]$,

and, from Eq. (16), the source $\tilde{\varrho}$ in momentum space:

$$
\begin{aligned}
\tilde{\varrho}(E, \mathbf{k})= & \frac{4}{\kappa^{2}}\left[\pi E^{2} \delta(E)+\frac{\sin (\pi p) \Gamma(2 p+1)}{t_{\mathrm{i}}^{2 p}|E|^{2 p-1}}\right] \\
& \times(2 \pi)^{3} \delta(\mathbf{k}) .
\end{aligned}
$$

For the form factor in Eq. (9), the fluctuation resulting from the integral (16) is

$\kappa h(t)=1-\left(\frac{2}{\Lambda t_{\mathrm{i}}}\right)^{2 p} \frac{\Gamma\left(\frac{1}{2}+p\right)}{\sqrt{\pi}}{ }_{1} F_{1}\left(-p ; \frac{1}{2} ;-\frac{1}{4} t^{2} \Lambda^{2}\right)$, where ${ }_{1} F_{1}$ is the confluent hypergeometric function of the first kind (Kummer's function):

${ }_{1} F_{1}(b ; c ; z):=\frac{\Gamma(c)}{\Gamma(b)} \sum_{l=0}^{+\infty} \frac{\Gamma(b+l)}{\Gamma(c+l)} \frac{z^{l}}{l !}$.

The transcendental equation (20) determines the value of $\Lambda t_{\mathrm{i}}$ such that $h\left(t_{\mathrm{i}}\right)=0$. This only affects unimportant time rescalings and the normalization of the scale factor

$$
a(t)=\left(\frac{2}{\Lambda t_{\mathrm{i}}}\right)^{p} \sqrt{\frac{\Gamma\left(\frac{1}{2}+p\right)}{\sqrt{\pi}}{ }_{1} F_{1}\left(-p ; \frac{1}{2} ;-\frac{1}{4} t^{2} \Lambda^{2}\right)} .
$$

The early- and late-time behavior can easily be found from the asymptotics of Kummer's function (e.g., [23]):

$$
\begin{aligned}
{ }_{1} F_{1}\left(b ; c ;-z^{2}\right) & \stackrel{z \rightarrow \pm \infty}{\sim} \frac{\Gamma(c)}{\Gamma(c-b)}\left(z^{2}\right)^{-b}, \\
{ }_{1} F_{1}\left(b ; c ;-z^{2}\right) & \stackrel{z \rightarrow 0}{\sim} 1 .
\end{aligned}
$$

We obtain

$$
\begin{aligned}
& a(t) \stackrel{t \rightarrow \pm \infty}{\sim}\left|\frac{t}{t_{\mathrm{i}}}\right|^{p}, \\
& a(t) \stackrel{t \rightarrow 0}{\sim} a_{*}:=\sqrt{\frac{\Gamma\left(\frac{1}{2}+p\right)}{\sqrt{\pi}}}\left(\frac{2}{\Lambda t_{\mathrm{i}}}\right)^{p} .
\end{aligned}
$$

The transition between these two regimes is set by the critical time $1 / \Lambda$. The general picture is that, at late times, the universe expands as a power law determined by its matter content. Common cases are radiation $(p=1 / 2)$, dust matter $(p=2 / 3)$, and acceleration-inducing components such as inflaton and quintessence $(p>1)$. In all these scenarios, there is a finite bounce at $t=0$, whose value increases with $p$ if $\Lambda t_{\mathrm{i}} \sim 1$. The most direct agent responsible for the bounce is asymptotic freedom: when the energy scale $\Lambda$ is sent to infinity, $a_{*} \rightarrow 0$.

Figures 1 and 2 illustrate the bouncing behavior of, respectively, the scale factor and the Ricci scalar $R=6(\ddot{a} / a+$ $\dot{a}^{2} / a^{2}$ ) for radiation and for a large- $p$ example. In the first case, we used the special form of Kummer's function ${ }_{1} F_{1}\left(-1 / 2 ; 1 / 2 ;-z^{2}\right)=\mathrm{e}^{-z^{2}}+\sqrt{\pi} z \operatorname{erf}(z)$, where erf is the error function:

$a(t)=\sqrt{\frac{2 \mathrm{e}^{-\frac{1}{4} \Lambda^{2} t^{2}}}{\sqrt{\pi} \Lambda t_{\mathrm{i}}}+\frac{t}{t_{\mathrm{i}}} \operatorname{erf}\left(\frac{\Lambda t}{2}\right) .}$

A consequence of Eq. (22) is that one always has superacceleration near the bounce independently of the value of $p$. This mechanism of super-inflation, which does not need any slow-rolling or exotic (e.g., phantom) scalar field, may be viewed as due to the vacuum energy associated with the graviton fluctuation, which is present for any type of matter content. In fact, in our model there is no ghost involved in the 

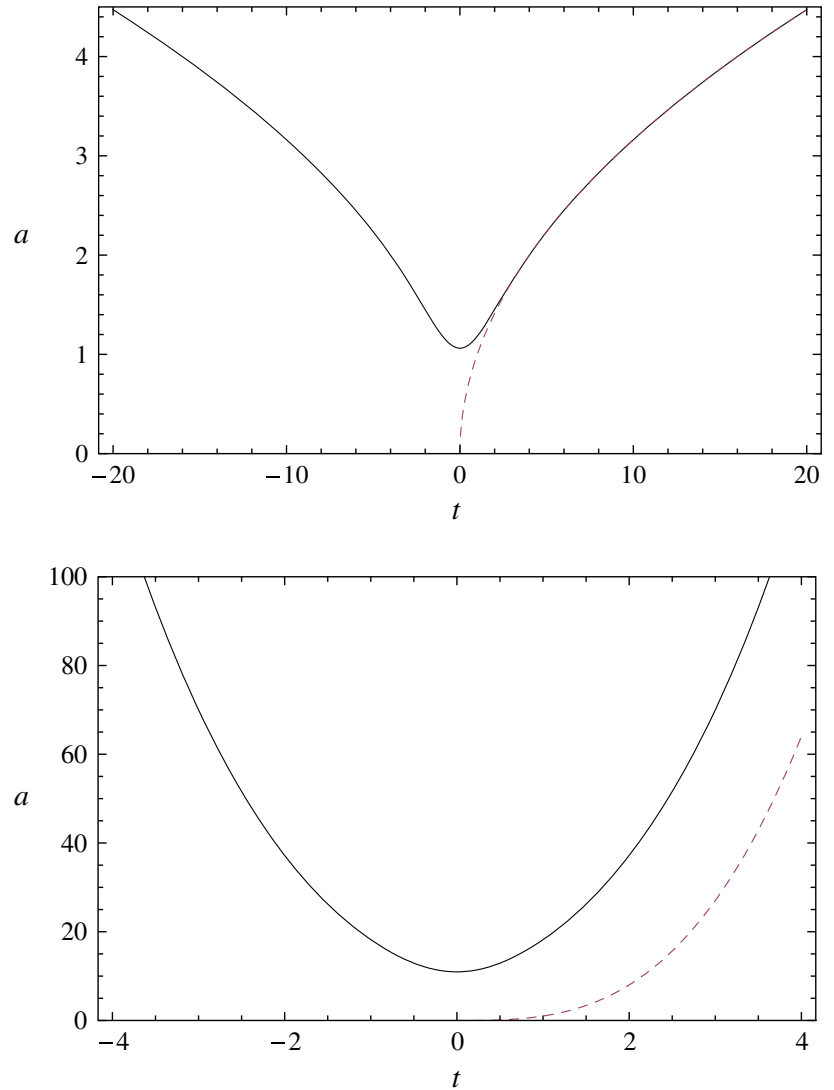

Fig. 1 Typical bouncing profile of the scale factor for $p<1$ (upper plot, $p=1 / 2$, radiation) and $p>1$ (lower plot, $p=3$, accelerating universe), for $\Lambda=1=t_{\mathrm{i}}$. The convexity of the curve changes sign at $p=1$. Dashed curves show the corresponding power-law profiles $a \sim t^{p}$ with big-bang singularity

regularization of the solution. The singularity removal is due only to the form of the graviton propagator and not to other physical or unphysical degrees of freedom. While any local higher-derivative theory of gravity typically shows ghostlike degrees of freedom, in the present case the non-local nature of the propagator gives a regular bouncing solution without introducing ghosts. Figure 3 shows positivity of the acceleration $\ddot{a}$, and a graceful exit from inflation when the matter content obeys the classical dominant energy condition (i.e., non-inflationary matter).

The Hubble parameter associated with the solution (22) is

$H(t)=\frac{\dot{a}}{a}=\frac{p \Lambda^{2} t}{2} \frac{{ }_{1} F_{1}\left(1-p ; \frac{3}{2} ;-\frac{1}{4} t^{2} \Lambda^{2}\right)}{{ }_{1} F_{1}\left(-p ; \frac{1}{2} ;-\frac{1}{4} t^{2} \Lambda^{2}\right)}$.

At the bounce, $H=0$. Asymptotically,

$H(t) \stackrel{t \rightarrow \pm \infty}{\sim} \frac{p}{t}, \quad H(t) \stackrel{t \rightarrow 0}{\sim} \frac{p \Lambda^{2}}{2} t$,

and, integrating at small times, we have

$a(t) \sim \exp \left(\frac{p}{4} \Lambda^{2} t^{2}\right) \quad$ as $\quad t \rightarrow 0$,
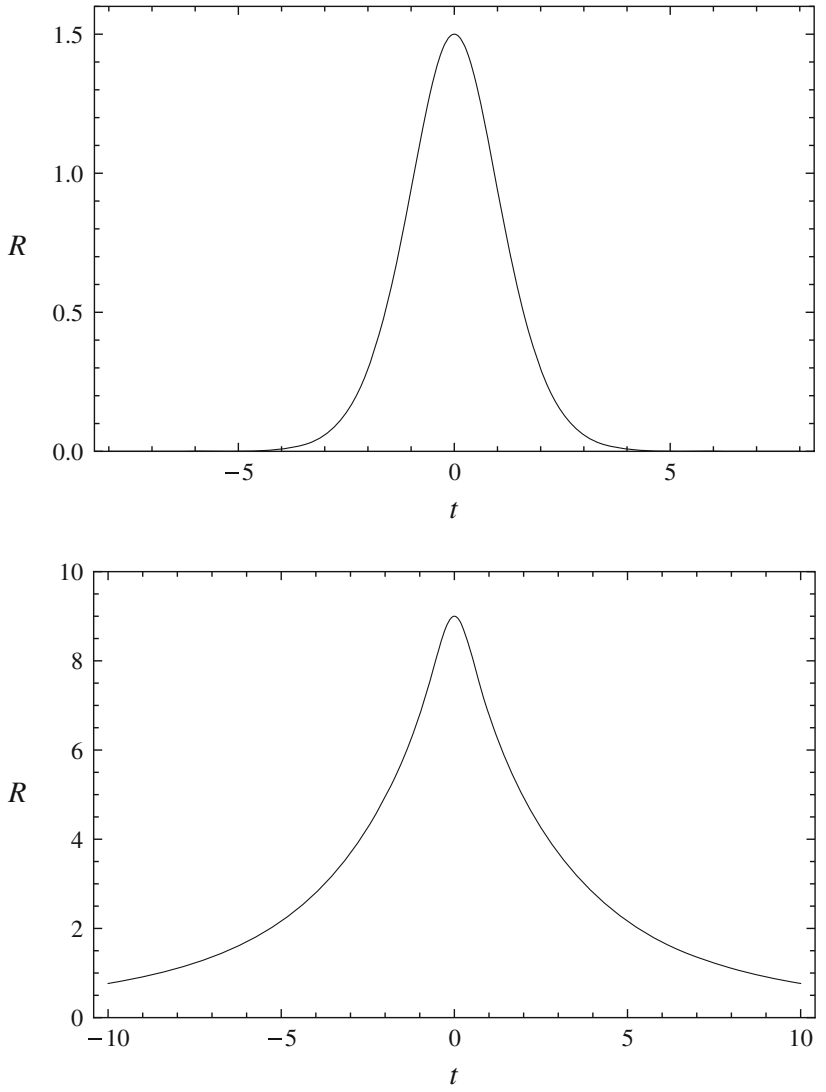

Fig. 2 Ricci scalar $R$ for $p=1 / 2$ (top) and $p=3$ (bottom). The curvature increases towards the bounce, where it acquires a non-singular value

thus getting the asymptotic behavior of the bounce in a neighborhood of $t=0$. The scale factor $a(t)$ for small times has a typical super-acceleration profile. This reminds one of an early contribution on cosmology in higher-derivative gravity [102], even if, as just stated, in our case we do not need to invoke the inflaton.

We conclude this section with three comments. First, we have not checked the stability of the profile (22). In the case of general non-polynomial theories, it is not obvious whether non-locality may trigger potentially dangerous instabilities. This is not so in the present case, where the non-local operator is the exponential of the d'Alembertian. There is reiterated evidence in the literature that such a strongly damping form factor actually improves any stability-related problem (exponential non-locality is under much greater control than other non-local models; see, e.g., [84,103]). Moreover, stability of the solution can be checked by looking at the behavior at early and late times separately (the complete perturbation would then be given by joining the two asymptotic behaviors). At late times, however, our solution reduces to standard powerlaw cosmology with standard dynamical equations, whose properties are well known. Therefore, one would need to verify stability only in the asymptotic-freedom regime. In 

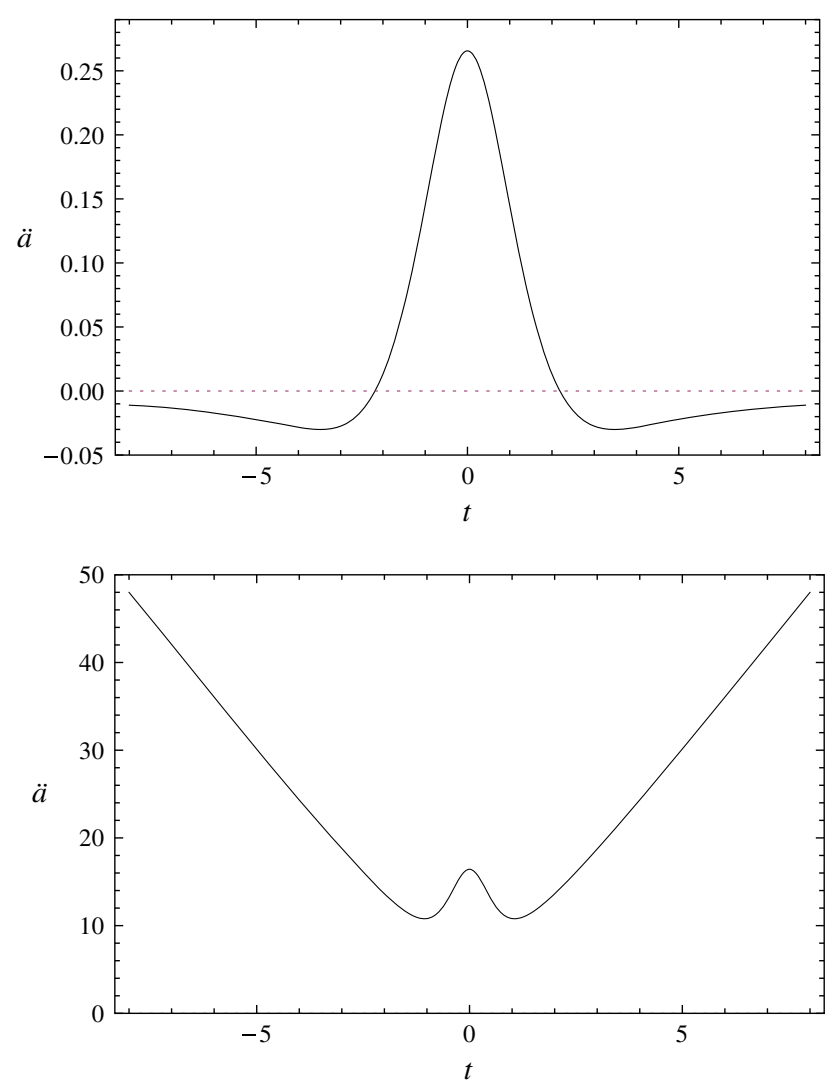

Fig. $3 \ddot{a}(t)$ for $p=1 / 2$ (top) and $p=3$ (bottom). In the presence of non-accelerating matter content (in this case, late-time radiation), when $\ddot{a}=0$ the (super-)inflationary era near the bounce naturally ends

this limit, however, the dynamical equations are linear in $a^{2}$ and the perturbation analysis becomes trivial. Therefore, the linear homogeneous perturbation $\delta\left(a^{2}\right) \approx 2 a(t) \delta a(t)$ of the solution $a(t)$ obeys the same linearized equation of the background (with unperturbed differential operators), $\delta a \propto a$, and the perturbed background $a+\delta a \propto a$ is only a physically irrelevant re-normalization of the scale factor. As a side remark, there should be no instability issues generated by non-locality even at the level of inhomogeneities. This is expected on the grounds that non-local theories with entire functions, such as exponential operators, do not introduce ghost, tachyon or Laplacian instabilities. We do not expect these arguments to be altered by a full calculation, which could be performed only with the knowledge of the full dynamics. This goes beyond the goals of the present paper.

Second, Kasner solutions are also straightforward. In ordinary Einstein-Hilbert gravity, the solution for a flat homogeneous anisotropic universe is $a_{i}(t)=\left|t / t_{\mathrm{i}}\right|^{p_{i}}$, where $i=1,2,3$ and the exponents $p_{i}$ obey the two conditions $\sum_{i} p_{i}=1=\sum_{i} p_{i}^{2}$. Following the above procedure (see [98] for Kasner solutions in a local quantum-gravity model), from the propagator we get three copies of Eq. (22). This formula is automatically well-defined for all the values of $p_{i}$

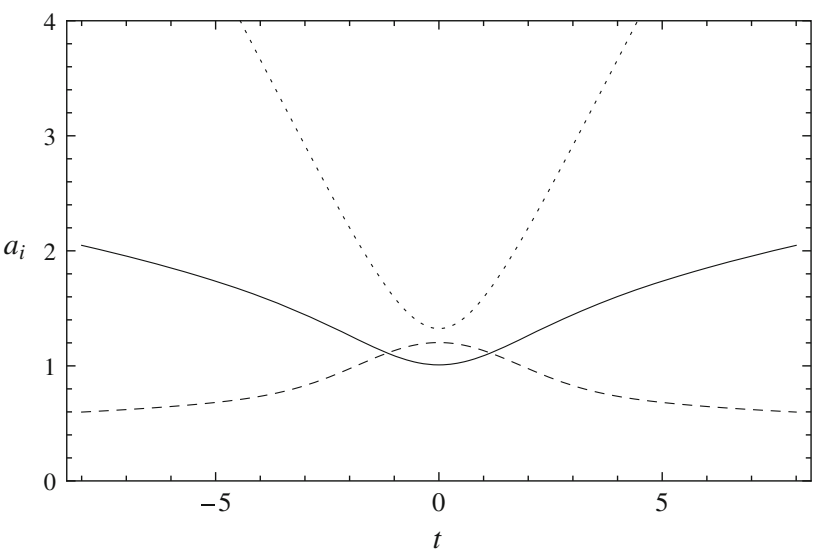

Fig. 4 The three scale factors $a_{i}(t)$ of a Kasner solution with $p_{1}=$ $-1 / 4$ (dashed curve), $p_{2}=(5-\sqrt{5}) / 8 \approx 0.35$ (solid curve), and $p_{3}=(5+\sqrt{5}) / 8 \approx 0.90($ dotted curve $)$, with $\Lambda=1=t_{\mathrm{i}}$

allowed by the Kasner conditions. Each scale factor reaches its local extremum (a minimum for two $p_{i}>0$ and a maximum for $p_{i}<0$ ) at different values dictated by Eq. (24). Going forwards in time towards $t=0$ and beyond, two directions contract, undergo a bounce, and begin to expand. In the meanwhile, the third direction expands from a minimum value $a_{i *}$, reaches a maximal extension at the bounce, and then contracts back to $a_{i *}$. Figure 4 shows one such solution.

Finally, we also checked that, in models where a Wick rotation is not necessary, the bounce picture persists. Let us recall that a Wick rotation was required because, in momentum space, the form factor (9) is not convergent when integrating in $k^{0}, V\left(k^{2}\right)=\exp \left(\Lambda^{2} k^{2}\right)=\exp \left[\Lambda^{2}\left(k_{0}^{2}-|\mathbf{k}|^{2}\right)\right]$. On the other hand, using even powers of the Laplace-Beltrami operator renders the form factor convergent without transforming to imaginary time, and the bouncing-accelerating scenario still holds. For instance, in Krasnikov's model with the operator $V(\square)=\mathrm{e}^{-\square^{2} / \Lambda^{4}}$, the solution with power-law asymptotic limit is a superposition of generalized hypergeometric functions ${ }_{q} F_{s}$, bounded from below (respectively, above) for $p>0(<0)$ by a symmetric bounce at some $a_{*} \neq 0$.

\subsection{Alternative derivation of the solution}

The solution (22) can also be found via the diffusion-equation method [82], which has proven to be a powerful tool both to address the Cauchy problem in exponential-type non-local systems [103] and to find non-perturbative tachyon solutions in string theory at the level of target actions $[25,82,104,105]$. While this method works well for matter fields on Minkowski background, the diffusion equation becomes non-linear when applied to the metric itself, since its solution appears also in the Laplace-Beltrami operator. For this reason, a particular gravitational action was constructed which allowed one 
to circumvent this problem [44]. Here, on the other hand, the action (8) gives rise to non-local, non-linear equations of motions for which the diffusion approach seems unsuitable. Fortunately, asymptotic freedom guarantees a window of applicability of the method in the limit where all interactions, including those of gravity with itself, are negligible.

Linearizing the action up to second order in perturbative fluctuations, one ends up with Eq. (6). Here, however, we do not invoke perturbation theory and throw away self- and matter interactions, but we encode them into an effective mass $m_{\text {eff }}$ for the gravitational field. The latter obeys the equation of motion $\square \mathrm{e}^{\square / \Lambda^{2}} h_{\mu \nu}+m_{\text {eff }}^{2} h_{\mu \nu}=0$. To solve this equation (or, approximately, its non-linear extensions), we promote $h_{\mu \nu}(x) \rightarrow h_{\mu \nu}(x, r)$ to a field living in $D+1$ dimensions, where $r$ is an artificial extra direction (dimensionally, a squared length), and assume that $h$ obeys the diffusion equation

$\left(\partial_{r}-\square\right) h_{\mu \nu}(x, r)=0, \quad h_{\mu \nu}(x, 0)=h_{\mu \nu}^{\mathrm{cl}}(x)$,

with a given set of initial conditions $h_{\mu \nu}^{\mathrm{cl}}(x)$. Here $\square$ is the Laplace-Beltrami operator in the background metric, i.e., $\square=\eta^{\mu \nu} \partial_{\mu} \partial_{\nu}$ in our case. Once the solution is found, the parameter $r$ is fixed at some constant value $r=r_{*}$ such that the equation of motion $\square h_{\mu \nu}\left(x, r_{*}+1 / \Lambda^{2}\right)+$ $m_{\text {eff }}^{2} h_{\mu \nu}\left(x, r_{*}\right)=0$ is solved.

For a homogeneous setting, the problem is drastically simplified. The only non-vanishing components of the metric are the diagonal spatial ones, and we need only to consider one diffusion equation for $a^{2}(t, r)$, with Laplace-Beltrami operator $\square=\partial_{t}^{2}$ (spatial derivatives are immaterial). The initial condition at $r=0$ is nothing but the asymptotic classical profile at $t \rightarrow \pm \infty$ because the solution only depends on the ratio $-t^{2} /(4 r)$ (this can be checked either a posteriori or beforehand by a simple scaling argument). Given a powerlaw initial condition, the solution of the diffusion equation

$\left(\partial_{r}-\partial_{t}^{2}\right) a^{2}(t, r)=0, \quad a^{2}(t, 0)=a_{\mathrm{cl}}^{2}(t)=\left|\frac{t}{t_{\mathrm{i}}}\right|^{2 p}$,

is, when evaluated at $r=r_{*}$, Eq. (22) with $\Lambda^{2}=1 / r_{*}$. The proof of this statement is essentially the calculation in [23] for a scalar-field profile in non-local cosmologies with exponential operators. ${ }^{4}$ Summarizing the procedure in a nutshell, one expands the initial condition $a^{2}(t, 0)$ as an integral superposition of the eigenfunctions $\mathrm{e}^{ \pm \mathrm{i} E t}$ of the exponential operator $V^{-1}(\square)$. Applying $V^{-1}(\square)$ to the initial condition and performing the integration, one obtains a linear combination $C_{1} a_{1}^{2}(t, r)+C_{2} a_{2}^{2}(t, r)$ of the two solutions to the

\footnotetext{
4 The reader can track down the steps in [103] from Eqs. (45) to (81), with the following mapping from the symbols used there to those adopted here: $\Psi=\Psi_{3} \rightarrow a^{2}, \phi_{0} \rightarrow t_{\mathrm{i}}^{-2 p}, \theta / 4 \rightarrow p$, $v \rightarrow 1 / 2, H_{0} \rightarrow 0, \alpha \rightarrow-1$. In [103], the opposite convention $\eta=\operatorname{diag}(-,+, \cdots,+)$ is used for the spacetime signature.
}

second-order equation (30), where

$$
\begin{aligned}
a_{1}^{2}(t, r)= & \frac{2 \Gamma(1+p)}{\sqrt{\pi}}\left(\frac{4 r}{t_{\mathrm{i}}^{2}}\right)^{p} \sqrt{\frac{t^{2}}{4 r}} \\
& \times{ }_{1} F_{1}\left(\frac{1}{2}-p ; \frac{3}{2} ;-\frac{t^{2}}{4 r}\right), \\
a_{2}^{2}(t, r)= & \left(-\frac{4 r}{t_{\mathrm{i}}^{2}}\right)^{p} \Psi\left(-p ; \frac{1}{2} ;-\frac{t^{2}}{4 r}\right),
\end{aligned}
$$

and $\Psi$ is the confluent hypergeometric function of the second kind. The solution $a_{1}^{2}$ has a big-bang singularity and is not well-defined (positive definite, finite, and so on) for all values of $p>0$; a special case is $p=1 / 2$, for which $a_{1}(t)=\sqrt{\left|t / t_{\mathrm{i}}\right|}$. The solution $a_{2}^{2}$ is complex-valued for general $p$; for $p=1$, however, we get $a_{2}(t)=\sqrt{2 r / t_{\mathrm{i}}^{2}+\left(t / t_{\mathrm{i}}\right)^{2}}$, which is real-valued and bouncing for $r \neq 0$, and singular if one takes trivial diffusion $(r=0$, also found in [57]). The choice of coefficients $C_{1}=-\mathrm{i} \tan (\pi p)$ and $C_{2}=\mathrm{e}^{-\mathrm{i} \pi p} / \cos (\pi p)$ gives (the square of) our solution (22). This linear combination is real-valued, big-bang free, superaccelerating near the bounce, and valid for all positive $p$. It is the latter criterion which makes us believe that bouncing solutions are typical in this theory, despite the existence of non-bouncing very special cases such as those above.

\subsection{Effective dynamics}

The solution (22) is approximate. Thanks to asymptotic freedom, it is valid at early times $t \lesssim t_{\mathrm{i}}$ where interactions are negligible. It is valid also at late times, where the theory reduces to ordinary Einstein-Hilbert gravity plus sub-leading quadratic terms (Stelle model). To check its viability outside these regimes, it is interesting to fit the Hubble parameter (26) with the effective energy density $\rho_{\text {eff }}$ defining the modified Friedmann equation (1). In the right-hand side of that equation, we take the energy density $\rho$ to be the one of a standard power-law cosmology, but with $a=\left|t / t_{\mathrm{i}}\right|^{p}$ replaced by Eq. (22),

$\rho(t)=\frac{3 p^{2}}{\kappa^{2} t_{\mathrm{i}}^{2}} \frac{1}{a^{2 / p}(t)}$,

while the critical energy density $\rho_{*} \neq \rho_{\text {eff }}(0)$ is fixed by the bounce scale factor $a_{*}=a(0)$ in (24),

$$
\begin{aligned}
\rho_{*} & =\frac{3 p^{2}}{\kappa^{2} t_{\mathrm{i}}^{2}} \frac{1}{a_{*}^{2 / p}}=\frac{3 p^{2} \Lambda^{2}}{4 \kappa^{2}}\left[\frac{\sqrt{\pi}}{\Gamma\left(\frac{1}{2}+p\right)}\right]^{\frac{1}{p}} \\
& =\frac{3 p^{2}}{32 \pi}\left[\frac{\sqrt{\pi}}{\Gamma\left(\frac{1}{2}+p\right)}\right]^{\frac{1}{p}} \frac{\Lambda^{2}}{m_{\mathrm{Pl}}^{2}} \rho_{\mathrm{Pl}},
\end{aligned}
$$

where $m_{\mathrm{Pl}}=\sqrt{\hbar c / G} \approx 1.2209 \times 10^{19} \mathrm{GeV}$ is the Planck mass and $\rho_{\mathrm{Pl}}=m_{\mathrm{Pl}}^{4} \approx 2.2 \times 10^{76} \mathrm{GeV}^{4}$ is the Planck energy 

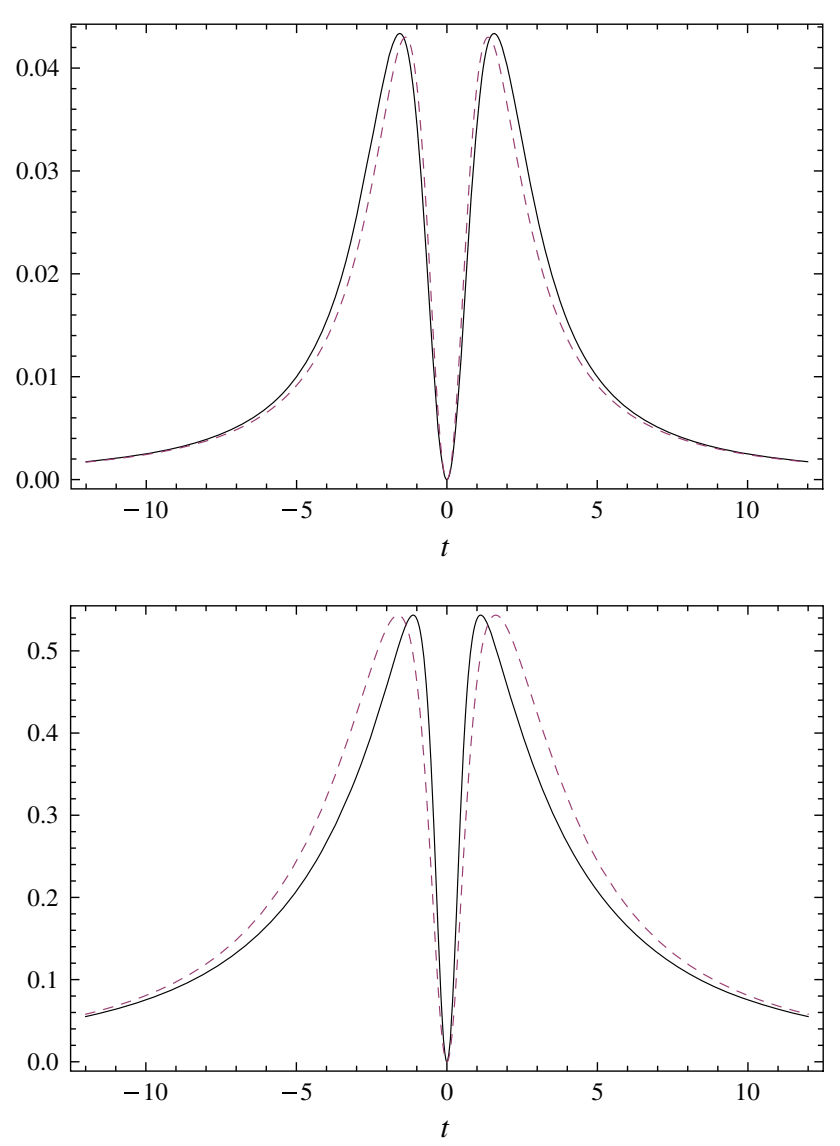

Fig. 5 Plot of $H^{2}$ (Eq. 26, solid curve) and the effective energy density (35) (dashed line), for $p=1 / 2, \beta=0.83$ (upper plot) and $p=3$, $\beta=1.3$ (lower plot), with $\Lambda=1$

density. Overall,

$$
\frac{\kappa^{2}}{3} \rho_{\mathrm{eff}}(t)=\frac{p^{2}}{t_{\mathrm{i}}^{2} a^{2 / p}(t)}\left\{1-\left[\frac{a_{*}}{a(t)}\right]^{\frac{2 \beta}{p}}\right\},
$$

which is the quantity plotted in Fig. 5. For $p=1 / 2$ (radiation case), we achieved a good qualitative fit with $\beta \approx 0.83$, while for $p=3$ we plot $\beta \approx 1.3$.

Notice that there may be no compelling reason to impose an all-scale fitting as in the figure. Using the asymptotic limits (23), we have Eq. (27) and

$$
\begin{aligned}
& \sqrt{\frac{\kappa^{2}}{3} \rho_{\mathrm{eff}}(t)} \stackrel{t \rightarrow \pm \infty}{\sim} \frac{p}{t}, \\
& \sqrt{\frac{\kappa^{2}}{3} \rho_{\mathrm{eff}}(t)} \stackrel{t \rightarrow 0}{\sim} \sqrt{\frac{2 \beta}{t_{\mathrm{i}}^{2} a_{*}^{2 / p} \Lambda^{2}}} \frac{p \Lambda^{2}}{2} t .
\end{aligned}
$$

The relative error at the origin is minimized for

$$
\beta=a_{*}^{\frac{2}{p}} \frac{\Lambda^{2} t_{\mathrm{i}}^{2}}{2}=2\left[\frac{\Gamma\left(\frac{1}{2}+p\right)}{\sqrt{\pi}}\right]^{\frac{1}{p}},
$$

equal to $\beta \approx 0.64$ for $p=1 / 2$ and $\beta \approx 2.47$ for $p=3$. For these values (which neither correspond to the fit of the figure nor to a least maximal error in the fit), there is a significant difference in the height of the local symmetric maxima at intermediate times. Conversely, in the plots the maximal relative error occurs at the origin. We believe that Eq. (37) better represents the asymptotic solutions, since higher-order curvature and quantum corrections are expected anyway to modify the evolution at mesoscopic scales, where the two lumps are located.

Assuming a standard Raychaudhuri equation $\dot{\rho}_{\text {eff }}+$ $3 H\left(P+\rho_{\text {eff }}\right)=0$, one can also obtain an effective pressure $P$ and barotropic index $w$,

$$
\begin{aligned}
P(t) & :=-\rho_{\mathrm{eff}}-\frac{\dot{\rho}_{\mathrm{eff}}}{3 H}=-\frac{1}{\kappa^{2}}\left(H^{2}+2 \frac{\ddot{a}}{a}\right), \\
w(t) & :=\frac{P}{\rho_{\mathrm{eff}}}
\end{aligned}
$$

which are depicted in Figs. 6 and 7, respectively. At the bounce, one has a non-vanishing finite effective pressure $P(0)=-R(0) /\left(3 \kappa^{2}\right)$, where $R(0)=3 p \Lambda^{2}$ is the Ricci scalar. At late times, $w \rightarrow-1+2 /(3 p)$, while at early times

$w(t) \stackrel{t \rightarrow 0}{\sim}-1-\frac{4}{3 p \Lambda^{2}} \frac{1}{t^{2}}$,

where one has super-acceleration $(w<-1)$.

\section{Conclusions}

In this paper, we have proposed a non-local model of gravity with improved UV behavior. We have focused only on its cosmology, and used the property of asymptotic freedom to find approximate solutions valid both at early and late times. In general, these solutions possess a bounce and avoid the big-bang singularity, and have an early era of acceleration with a natural exit in the absence of inflaton fields. Specifically, the universe is characterized by a superacceleration regime at the bounce, with effective barotropic index $w<-1$.

Since we have not solved the full equations of motion exactly, we have bypassed the problem of getting the dynamics of a theory with all sectors (both gravity and matter) being non-local. Instead, we have matched the solution (22) with an effective dynamics encoded in the modified Friedmann equation (1). The good agreement between this solution and Eq. (1) at all scales suggests that the problem is not unsolvable. The diffusion-equation method, applied in Sect. 3.3 to find an alternative derivation of the profile (22), might be a useful tool in this respect. Intriguingly, Eq. (30) acts as a "beta function," determining the running of the metric with the cut-off length scale $1 / \Lambda$, which plays the role of diffusion time $\sim \sqrt{r}$. The possibility to study the dynamics 

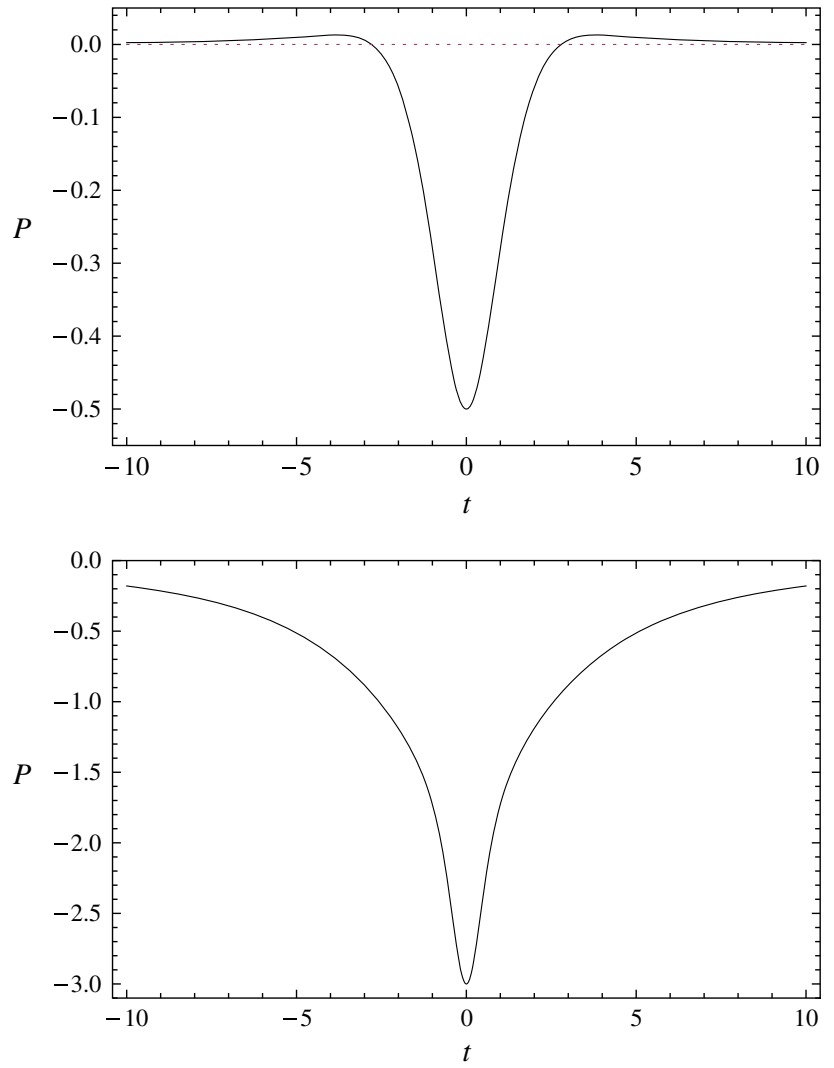

Fig. 6 The effective pressure $P(t)=-\rho_{\text {eff }}-\dot{\rho}_{\text {eff }} /(3 H)$ for $p=1 / 2$ (top) and $p=3$ (bottom). At the bounce, it tends to a non-zero finite value $P(0)=-R(0) /\left(3 \kappa^{2}\right)$. Here we set $\kappa^{2}=1=\Lambda$

of this class of non-local theories via the diffusion method is a direct consequence of their renormalization properties [82].

It is remarkable that the bouncing dynamics of the present model can be reproduced semi-quantitatively by an effective equation with only one free parameter. The value of the critical energy density $\rho_{*}$ is also plausible. From Eq. (34) and setting the cut-off to its natural value $\Lambda=m_{\mathrm{Pl}}$, we get $\rho_{*} \approx 0.02 \rho_{\mathrm{Pl}}$ for $p=1 / 2$ and $\rho_{*} \approx 0.22 \rho_{\mathrm{Pl}}$ for $p=3$. Models such that $\rho \leq \rho_{*} \leq \rho_{\text {Pl }}$ must have $p \leq p_{\max } \approx 12.67$. For $p>p_{\max }$, the critical energy density exceeds $\rho_{\mathrm{Pl}}$, and the energy density of the universe can become trans-Planckian near and at the bounce. Therefore, scenarios with too-large $p$ are not well represented by Eq. (1). This is not an issue in our model, since we have early-universe acceleration by default also for small values of $p$.

The relative error between the approximate solution $H^{2}$ and the effective energy density $\kappa^{2} \rho_{\text {eff }} / 3$ could be reduced by a more refined Ansatz for the effective Friedmann equation. Further study of this method may turn out to shed some light on the exact dynamics, which should be developed in parallel starting from the actual equations of motion. For
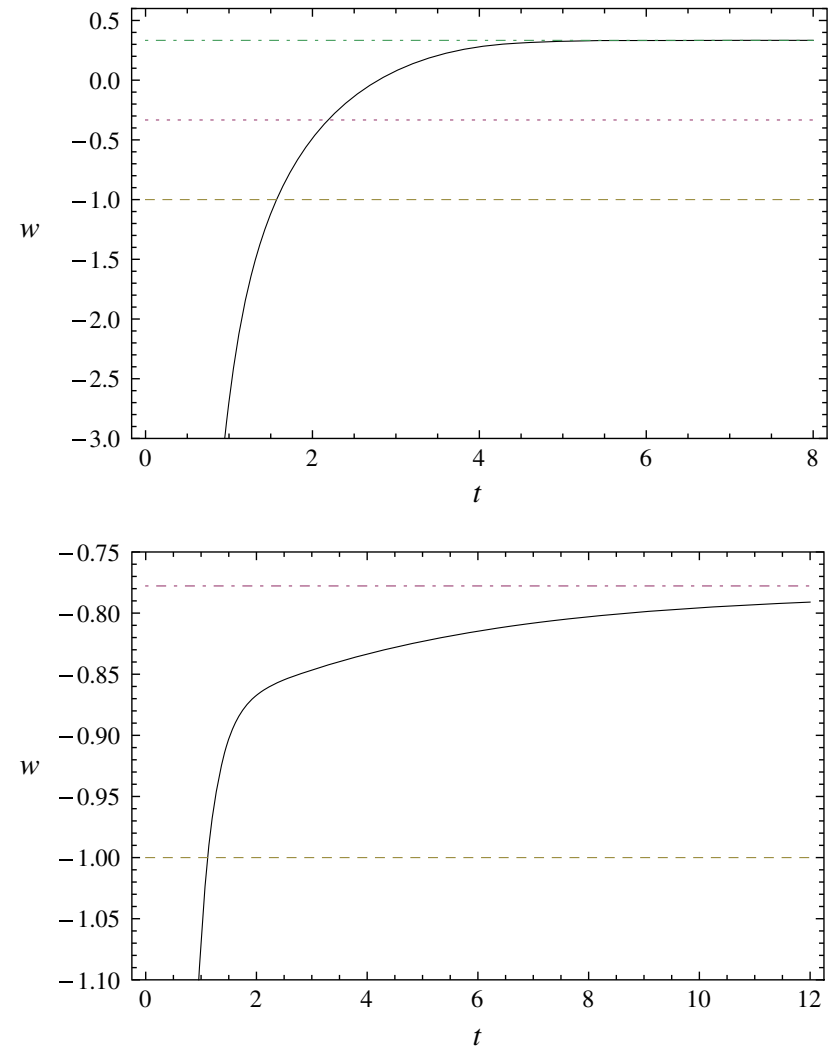

Fig. 7 The effective barotropic index $w(t)=-P(t) / \rho_{\text {eff }}(t)$ for $p=$ $1 / 2$ (top) and $p=3$ (bottom), with $\Lambda=1$. For $w<-1 / 3$, one has acceleration and for $w<-1$ (small times) super-acceleration, in accordance with Eq. (28). The lines $w=-1+2 /(3 p)$ (dot-dashed), $w=-1 / 3($ dotted $)$ and $w=-1$ (dashed) are shown for reference

instance, once derived the actual equations of motion of the theory one could plug the solution $a(t)$ found here, and check whether a reasonable matter sector is recovered. In particular, from our modified Kasner solution it should be possible to check whether some form of BKL chaos survives in the full anisotropic dynamics.

This should not only clarify whether the bounce picture is robust in our theory, but also to which class of singularityfree cosmologies the model belongs to. As an exact dynamical equation, expression (1) with $\beta=1$ appears also in braneworlds with a timelike extra direction [106] and in purely homogeneous loop quantum cosmology (for reviews consult, e.g., $[107,108])$ only in the parameter choice for the so-called "improved" mini-superspace dynamics $[109,110]$ (for other parametrizations, Eq. (1) with $\beta=1$ no longer holds [111]). Although there is no relation between our framework and these high-energy cosmological models, they all share the same type of bounce where the right-hand side of the Friedmann equation receives a negative higher-order correction in the energy density. On the other hand, there is a different class of models where the correction is of the "dark radiation" form $-1 / a^{4}$, which is responsible for the 
bounce at $H=0$. Such is the case for the Randall-Sundrum braneworld with a spacelike extra dimension [112], HořavaLifshitz gravity without detailed balance $[113,114]$, and cosmologies with fermionic condensates $[115,116]$. As far as we pushed the analysis in this paper, the present model apparently lies in the first category, with the added bonus that we have an alternative mechanism of inflation of purely geometric origin.

Acknowledgments The authors thank G. Mena Marugán for comments on the manuscript and are grateful to J. Moffat for early discussions on related topics. G.C. and L.M. acknowledge the i-Link cooperation programme of CSIC (project ID i-Link0484) for partial sponsorship. The work of G.C. is under a Ramón y Cajal tenure-track contract; he also thanks Fudan University for the kind hospitality during the completion of this article. The work of P.N. has been supported by the German Research Foundation (DFG) grant NI 1282/2-1, and partially by the Helmholtz International Center for FAIR within the framework of the LOEWE program (Landesoffensive zur Entwicklung Wissenschaftlich-Ökonomischer Exzellenz) launched by the State of Hesse and by the European COST action MP0905 "Black Holes in a Violent Universe".

Open Access This article is distributed under the terms of the Creative Commons Attribution License which permits any use, distribution, and reproduction in any medium, provided the original author(s) and the source are credited.

Funded by $\mathrm{SCOAP}^{3}$ / License Version CC BY 4.0.

\section{References}

1. K. Ohmori, A review on tachyon condensation in open string field theories, hep-th/0102085

2. A. Sen, Tachyon dynamics in open string theory. Int. J. Mod. Phys. A 20, 5513 (2005). hep-th/0410103

3. E. Fuchs, M. Kroyter, Analytical solutions of open string field theory. Phys. Rep. 502, 89 (2011). arXiv:0807.4722

4. Y. Okawa, Analytic methods in open string field theory. Prog. Theor. Phys. 128, 1001 (2012)

5. P.G.O. Freund, M. Olson, Nonarchimedean strings. Phys. Lett. B 199, 186 (1987). doi:10.1016/0370-2693(87)91356-6

6. P.G.O. Freund, E. Witten, Adelic string amplitudes. Phys. Lett. B 199, 191 (1987). doi:10.1016/0370-2693(87)91357-8

7. L. Brekke, P.G.O. Freund, M. Olson, E. Witten, Nonarchimedean string dynamics. Nucl. Phys. B 302, 365 (1988). doi:10.1016/ 0550-3213(88)90207-6

8. V.S. Vladimirov, Ya.I. Volovich, On the nonlinear dynamical equation in the $p$-adic string theory. Theor. Math. Phys. 138, 297 (2004). doi:10.1023/B:TAMP.0000018447.02723.29. math-ph/0306018

9. V. Vladimirov, Nonlinear equations for $p$-adic open, closed, and open-closed strings. Theor. Math. Phys. 149, 1604 (2006). doi:10. 1007/s11232-006-0144-z. arXiv:0705.4600

10. T. Biswas, J.A.R. Cembranos, J.I. Kapusta, Thermal duality and Hagedorn transition from $p$-adic strings. Phys. Rev. Lett. 104, 021601 (2010). doi:10.1103/PhysRevLett.104.021601. arXiv:0910.2274

11. I. Ya, Aref'eva, Nonlocal string tachyon as a model for cosmological dark energy. AIP Conf. Proc. 826, 301 (2006). doi:10.1063/ 1.2193132. astro-ph/0410443
12. I.Ya. Aref'eva, L.V. Joukovskaya, Time lumps in nonlocal stringy models and cosmological applications, JHEP 0510, 087 (2005). doi:10.1088/1126-6708/2005/10/087. hep-th/0504200

13. I. Ya, Aref'eva, A.S. Koshelev, S. Yu. Vernov, Stringy dark energy model with cold dark matter. Phys. Lett. B 628, 1 (2005). doi:10. 1016/j.physletb.2005.09.017. astro-ph/0505605

14. G. Calcagni, Cosmological tachyon from cubic string field theory. JHEP 0605, 012 (2006). doi:10.1088/1126-6708/2006/05/ 012. hep-th/0512259

15. I.Ya. Aref'eva, A.S. Koshelev, Cosmic acceleration and crossing of $w=-1$ barrier from cubic superstring field theory, JHEP 0702, 041 (2007). doi:10.1088/1126-6708/2007/02/041. hep-th/0605085

16. I. Ya, Aref'eva, I.V. Volovich, On the null energy condition and cosmology. Theor. Math. Phys. 155, 503 (2008). doi:10.1007/ s11232-008-0041-8. hep-th/0612098

17. N. Barnaby, T. Biswas, J.M. Cline, $p$-adic inflation. JHEP 0704, 056 (2007). doi:10.1088/1126-6708/2007/04/056. hep-th/0612230

18. A.S. Koshelev, Non-local SFT tachyon and cosmology. JHEP 0704, 029 (2007). doi:10.1088/1126-6708/2007/04/029. hep-th/0701103

19. I.Ya. Aref'eva, L.V. Joukovskaya, S. Yu. Vernov, Bouncing and accelerating solutions in nonlocal stringy models, JHEP 0707, 087 (2007). doi:10.1088/1126-6708/2007/07/087. hep-th/0701184

20. I.Ya. Aref'eva, I.V. Volovich, Quantization of the Riemann zetafunction and cosmology, Int. J. Geom. Methods Mod. Phys. 4, 881 (2007). doi:10.1142/S021988780700234X. hep-th/0701284

21. J.E. Lidsey, Stretching the inflaton potential with kinetic energy. Phys. Rev. D 76, 043511 (2007). doi:10.1103/PhysRevD.76. 043511. hep-th/0703007

22. N. Barnaby, J.M. Cline, Large nongaussianity from nonlocal inflation. JCAP 0707, 017 (2007). doi:10.1088/1475-7516/2007/07/ 017. arXiv:0704.3426

23. G. Calcagni, M. Montobbio, G. Nardelli, Route to nonlocal cosmology. Phys. Rev. D 76, 126001 (2007). doi:10.1103/PhysRevD. 76.126001. arXiv:0705.3043

24. L.V. Joukovskaya, Dynamics in nonlocal cosmological models derived from string field theory. Phys. Rev. D 76, 105007 (2007). doi:10.1103/PhysRevD.76.105007. arXiv:0707.1545

25. G. Calcagni, G. Nardelli, Nonlocal instantons and solitons in string models. Phys. Lett. B 669, 102 (2008). doi:10.1016/j. physletb.2008.09.016. arXiv:0802.4395

26. L. Joukovskaya, Rolling solution for tachyon condensation in open string field theory, arXiv:0803.3484

27. I.Ya. Aref'eva, A.S. Koshelev, Cosmological signature of tachyon condensation. JHEP 0809, 068 (2008). arXiv:0804.3570

28. L. Joukovskaya, Dynamics with infinitely many time derivatives in Friedmann-Robertson-Walker background and rolling tachyons. JHEP 0902, 045 (2009). doi:10.1088/1126-6708/2009/ 02/045. arXiv:0807.2065

29. N. Barnaby, N. Kamran, Dynamics with infinitely many derivatives: variable coefficient equations. JHEP 0812, 022 (2008). doi:10.1088/1126-6708/2008/12/022. arXiv:0809.4513

30. N.J. Nunes, D.J. Mulryne, Non-linear non-local cosmology. AIP Conf. Proc. 1115, 329 (2009). doi:10.1063/1.3131521. arXiv:0810.5471

31. A.S. Koshelev, S. Yu. Vernov, Cosmological perturbations in SFT inspired non-local scalar field models. Eur. Phys. J. C 72, 2198 (2012). doi:10.1140/epjc/s10052-012-2198-4. arXiv:0903.5176

32. G. Calcagni, G. Nardelli, Cosmological rolling solutions of nonlocal theories. Int. J. Mod. Phys. D 19, 329 (2010). doi:10.1142/ S0218271810016440. arXiv:0904.4245

33. S. Yu. Vernov, Localization of non-local cosmological models with quadratic potentials in the case of double roots. Class. 
Quantum Grav. 27, 035006 (2010). doi:10.1088/0264-9381/27/ 3/035006. arXiv:0907.0468

34. S. Yu. Vernov, Localization of the SFT inspired nonlocal linear models and exact solutions. Phys. Part. Nucl. Lett. 8, 310 (2011). doi:10.1134/S1547477111030228. arXiv: 1005.0372

35. A.S. Koshelev, S. Yu. Vernov, Analysis of scalar perturbations in cosmological models with a non-local scalar field. Class. Quantum Grav. 28, 085019 (2011). doi:10.1088/0264-9381/28/ 8/085019. arXiv:1009.0746

36. N.V. Krasnikov, Nonlocal gauge theories. Theor. Math. Phys. 73, 1184 (1987). doi:10.1007/BF01017588. http://www.mathnet.ru/ php/archive.phtml? wshow=paper\&jrnid $=$ tmf $\&$ paperid $=5624 \&$ option_lang=eng [Teor. Mat. Fiz. 73, 235 (1987)]

37. E.T. Tomboulis, Superrenormalizable gauge and gravitational theories, hep-th/9702146

38. N. Arkani-Hamed, S. Dimopoulos, G. Dvali, G. Gabadadze, Nonlocal modification of gravity and the cosmological constant problem, hep-th/0209227

39. A.O. Barvinsky, Nonlocal action for long distance modifications of gravity theory. Phys. Lett. B 572, 109 (2003). doi:10.1016/j. physletb.2003.08.055. hep-th/0304229

40. A.O. Barvinsky, On covariant long-distance modifications of Einstein theory and strong coupling problem. Phys. Rev. D 71, 084007 (2005). doi:10.1103/PhysRevD.71.084007. hep-th/0501093

41. H.W. Hamber, R.M. Williams, Nonlocal effective gravitational field equations and the running of Newton's $G$. Phys. Rev. D 72, 044026 (2005). doi:10.1103/PhysRevD.72.044026. hep-th/0507017

42. T. Biswas, A. Mazumdar, W. Siegel, Bouncing universes in string-inspired gravity. JCAP 0603, 009 (2006). doi:10.1088/ 1475-7516/2006/03/009. hep-th/0508194

43. J. Khoury, Fading gravity and self-inflation. Phys. Rev. D 76, 123513 (2007). doi:10.1103/PhysRevD.76.123513. hep-th/0612052

44. G. Calcagni, G. Nardelli, Nonlocal gravity and the diffusion equation. Phys. Rev. D 82, 123518 (2010). doi:10.1103/PhysRevD.82. 123518. arXiv: 1004.5144

45. T. Biswas, T. Koivisto, A. Mazumdar, Towards a resolution of the cosmological singularity in non-local higher derivative theories of gravity. JCAP 1011, 008 (2010). doi:10.1088/1475-7516/2010/ 11/008. arXiv: 1005.0590

46. A.O. Barvinsky, Dark energy and dark matter from nonlocal ghost-free gravity theory. Phys. Lett. B 710, 12 (2012). doi:10. 1016/j.physletb.2012.02.075. arXiv:1107.1463

47. L. Modesto, Super-renormalizable quantum gravity. Phys. Rev. D 86, 044005 (2012). doi:10.1103/PhysRevD.86.044005. arXiv: 1107.2403

48. T. Biswas, E. Gerwick, T. Koivisto, A. Mazumdar, Towards singularity and ghost free theories of gravity. Phys. Rev. Lett. 108, 031101 (2012). doi:10.1103/PhysRevLett.108.031101. arXiv: 1110.5249

49. A.S. Koshelev, Modified non-local gravity, Rom. J. Phys. 57, 894 (2012). arXiv:1112.6410. http://www.nipne.ro/rjp/2012_57_5-6. html

50. L. Modesto, Super-renormalizable higher-derivative quantum gravity, arXiv: 1202.0008

51. A.S. Koshelev, SYu. Vernov, On bouncing solutions in nonlocal gravity. Phys. Part. Nucl. 43, 666 (2012). doi:10.1134/ S106377961205019X. arXiv:1202.1289

52. S. Alexander, A. Marcianò, L. Modesto, The hidden quantum groups symmetry of super-renormalizable gravity. Phys. Rev. D 85, 124030 (2012). doi:10.1103/PhysRevD.85.124030. arXiv: 1202.1824

53. L. Modesto, Super-renormalizable multidimensional quantum gravity: theory and applications. Astron. Rev. 8.2, 4 (2013). arXiv: 1202.3151
54. L. Modesto, Towards a finite quantum supergravity, arXiv: 1206.2648

55. T. Biswas, A.S. Koshelev, A. Mazumdar, S. Yu. Vernov, Stable bounce and inflation in non-local higher derivative cosmology. JCAP 1208, 024 (2012). doi:10.1088/1475-7516/2012/08/024. arXiv: 1206.6374

56. F. Briscese, A. Marcianò, L. Modesto, E.N. Saridakis, Inflation in (super-)renormalizable gravity. Phys. Rev. D 87, 083507 (2013). doi:10.1103/PhysRevD.87.083507. arXiv:1212.3611

57. A.S. Koshelev, Stable analytic bounce in non-local EinsteinGauss-Bonnet cosmology. Class. Quantum Grav. 30, 155001 (2013). doi:10.1088/0264-9381/30/15/155001. arXiv:1302.2140

58. B. Hasslacher, E. Mottola, Asymptotically free quantum gravity and black holes. Phys. Lett. B 99, 221 (1981). doi:10.1016/ 0370-2693(81)91112-6

59. M. Saadi, B. Zwiebach, Closed string field theory from polyhedra. Ann. Phys. 192, 213 (1989). doi:10.1016/0003-4916(89)90126-7

60. T. Kugo, H. Kunitomo, K. Suehiro, Nonpolynomial closed string field theory. Phys. Lett. B 226, 48 (1989). doi:10.1016/ 0370-2693(89)90287-6

61. T. Kugo, K. Suehiro, Nonpolynomial closed string field theory: action and its gauge invariance. Nucl. Phys. B 337, 434 (1990). doi:10.1016/0550-3213(90)90277-K

62. V.A. Kostelecký, S. Samuel, Collective physics in the closed bosonic string. Phys. Rev. D 42, 1289 (1990). doi:10.1103/ PhysRevD.42.1289

63. B. Zwiebach, Closed string field theory: quantum action and the B-V master equation. Nucl. Phys. B 390, 33 (1993). doi:10.1016/ 0550-3213(93)90388-6. hep-th/9206084

64. A. Sen, B. Zwiebach, A proof of local background independence of classical closed string field theory. Nucl. Phys. B 414, 649 (1994). doi:10.1016/0550-3213(94)90258-5. hep-th/9307088

65. A. Sen, B. Zwiebach, Quantum background independence of closed string field theory. Nucl. Phys. B 423, 580 (1994). doi:10. 1016/0550-3213(94)90145-7. hep-th/9311009

66. A. Sen, B. Zwiebach, A note on gauge transformations in BatalinVilkovisky theory. Phys. Lett. B 320, 29 (1994). doi:10.1016/ 0370-2693(94)90819-2. hep-th/9309027

67. Y. Okawa, B. Zwiebach, Twisted tachyon condensation in closed string field theory. JHEP 0403, 056 (2004). doi:10.1088/ 1126-6708/2004/03/056. hep-th/0403051

68. H. Yang, B. Zwiebach, Dilaton deformations in closed string field theory. JHEP 0505, 032 (2005). doi:10.1088/1126-6708/2005/ 05/032. hep-th/0502161

69. H. Yang, B. Zwiebach, A closed string tachyon vacuum? JHEP 0509, 054 (2005). doi:10.1088/1126-6708/2005/09/054. hep-th/0506077

70. Y. Michishita, Field redefinitions, $T$-duality and solutions in closed string field theories. JHEP 0609, 001 (2006). doi:10.1088/ 1126-6708/2006/09/001. hep-th/0602251

71. N. Moeller, Closed bosonic string field theory at quintic order: five-tachyon contact term and dilaton theorem. JHEP 0703, 043 (2007). doi:10.1088/1126-6708/2007/03/043. hep-th/0609209

72. N. Moeller, Closed bosonic string field theory at quintic order. II: marginal deformations and effective potential. JHEP 0709, 118 (2007). doi:10.1088/1126-6708/2007/09/118. arXiv:0705.2102

73. N. Moeller, A tachyon lump in closed string field theory. JHEP 0809, 056 (2008). doi:10.1088/1126-6708/2008/09/056. arXiv:0804.0697

74. S. Nojiri, S.D. Odintsov, Modified non-local- $F(R)$ gravity as the key for the inflation and dark energy. Phys. Lett. B 659, 821 (2008). doi:10.1016/j.physletb.2007.12.001. arXiv:0708.0924

75. S. Capozziello, E. Elizalde, S. Nojiri, S.D. Odintsov, Accelerating cosmologies from non-local higher-derivative gravity. Phys. Lett. B 671, 193 (2009). doi:10.1016/j.physletb.2008.11.060. arXiv:0809.1535 
76. S.I. Nojiri, S.D. Odintsov, M. Sasaki, Y.-L. Zhang, Screening of cosmological constant in non-local gravity. Phys. Lett. B 696, 278 (2011). doi:10.1016/j.physletb.2010.12.035. arXiv:1010.5375

77. Y.-L. Zhang, M. Sasaki, Screening of cosmological constant in non-local cosmology. Int. J. Mod. Phys. D 21, 1250006 (2012). doi:10.1142/S021827181250006X. arXiv:1108.2112

78. E. Elizalde, E.O. Pozdeeva, S. Yu. Vernov, Y.-L. Zhang, Cosmological solutions of a nonlocal model with a perfect fluid. JCAP 1307, 034 (2013). doi:10.1088/1475-7516/2013/07/034. arXiv: 1302.4330

79. S. Alexander, R. Brandenberger, J. Magueijo, Noncommutative inflation. Phys. Rev. D 67, 081301 (2003). doi:10.1103/ PhysRevD.67.081301. hep-th/0108190

80. M. Rinaldi, A new approach to non-commutative inflation. Class. Quantum Grav. 28, 105022 (2011). doi:10.1088/0264-9381/28/ 10/105022. arXiv:0908.1949

81. T. Biswas, A. Mazumdar, Super-inflation, non-singular bounce, and low multipoles. Class. Quantum Grav. 31, 025019 (2014). doi:10.1088/0264-9381/31/2/025019. arXiv:1304.3648

82. G. Calcagni, G. Nardelli, String theory as a diffusing system. JHEP 1002, 093 (2010). doi:10.1007/JHEP02(2010)093. arXiv:0910.2160

83. G. Calcagni, L. Modesto, Nonlocality in string theory. J. Phys. A 47, 355402 (2014). doi:10.1088/1751-8113/47/35/355402

84. N. Barnaby, N. Kamran, Dynamics with infinitely many derivatives: the initial value problem. JHEP 0802, 008 (2008). arXiv:0709.3968

85. G.V. Efimov, Nonlocal Interactions of Quantized Fields (in Russian) (Nauka, Moscow, 1977)

86. A. Smailagic, E. Spallucci, Lorentz invariance, unitarity in UVfinite of QFT on noncommutative spacetime. J. Phys. A 37, 7169 (2004). doi:10.1088/0305-4470/37/28/008. hep-th/0406174

87. E. Spallucci, A. Smailagic, P. Nicolini, Trace anomaly in quantum spacetime manifold. Phys. Rev. D 73, 084004 (2006). doi:10. 1103/PhysRevD.73.084004. hep-th/0604094

88. P. Nicolini, M. Rinaldi, A minimal length versus the Unruh effect. Phys. Lett. B 695, 303 (2011). doi:10.1016/j.physletb.2010.10. 051. arXiv:0910.2860

89. M. Kober, P. Nicolini, Minimal scales from an extended Hilbert space. Class. Quantum Grav. 27, 245024 (2010). doi:10.1088/ 0264-9381/27/24/245024. arXiv: 1005.3293

90. M. Asorey, J.L. López, I.L. Shapiro, Some remarks on high derivative quantum gravity. Int. J. Mod. Phys. A 12, 5711 (1997). doi:10. 1142/S0217751X97002991. hep-th/9610006

91. L. Modesto, J.W. Moffat, P. Nicolini, Black holes in an ultraviolet complete quantum gravity. Phys. Lett. B 695, 397 (2011). doi:10. 1016/j.physletb.2010.11.046. arXiv:1010.0680

92. L. Modesto, Finite quantum gravity, arXiv:1305.6741

93. G. Calcagni, L. Modesto, Proposal for field M-theory, arXiv: 1404.2137

94. M.J. Duff, D.J. Toms, Kaluza-Klein-Kounterterms, in Unification of Fundamental Particle Interactions II, ed. by J. Ellis, S. Ferrara (Springer, Amsterdam, 1983). doi:10.1007/ 978-1-4615-9299-0_3

95. K.S. Stelle, Renormalization of higher-derivative quantum gravity. Phys. Rev. D 16, 953 (1977). doi:10.1103/PhysRevD.16.953

96. M.J. Duff, Quantum corrections to the Schwarzschild solution. Phys. Rev. D 9, 1837 (1974). doi:10.1103/PhysRevD.9.1837

97. B. Broda, One-loop quantum gravity repulsion in the early Universe. Phys. Rev. Lett. 106, 101303 (2011). doi:10.1103/ PhysRevLett.106.101303. arXiv:6257
98. B. Broda, Quantum gravity stability of isotropy in homogeneous cosmology. Phys. Lett. B 704, 655 (2011). doi:10.1016/j.physletb. 2011.09.087. arXiv:1107.3468

99. C. Bambi, D. Malafarina, L. Modesto, Terminating black holes in quantum gravity. Eur. Phys. J. C 74, 2767 (2014). doi:10.1140/ epjc/s10052-014-2767-9. arXiv:1306.1668

100. A. Accioly, A. Azeredo, H. Mukai, Propagator, tree-level unitarity and effective nonrelativistic potential for higher-derivative gravity theories in $D$ dimensions. J. Math. Phys. 43, 473 (2002). doi:10. $1063 / 1.1415743$

101. P. Van Nieuwenhuizen, On ghost-free tensor Lagrangians and linearized gravitation. Nucl. Phys. B 60, 478 (1973). doi:10.1016/ 0550-3213(73)90194-6

102. M.D. Pollock, On super-exponential inflation in a higherdimensional theory of gravity with higher-derivative terms. Nucl. Phys. B 309, 513 (1988). doi:10.1016/ 0550-3213(88)90456-7 (erratum ibid. B 374, 469 (1992). doi:10.1016/0550-3213(92)90363-G)

103. G. Calcagni, M. Montobbio, G. Nardelli, Localization of nonlocal theories. Phys. Lett. B 662, 285 (2008). arXiv:0712.2237

104. G. Calcagni, G. Nardelli, Tachyon solutions in boundary and cubic string field theory. Phys. Rev. D 78, 126010 (2008). doi:10.1103/ PhysRevD.78.126010. arXiv:0708.0366

105. G. Calcagni, G. Nardelli, Kinks of open superstring field theory. Nucl. Phys. B 823, 234 (2009). doi:10.1016/j.nuclphysb.2009.08. 004. arXiv:0904.3744

106. Y. Shtanov, V. Sahni, Bouncing brane worlds. Phys. Lett. B 557, 1 (2003). doi:10.1016/S0370-2693(03)00179-5. gr-qc/0208047

107. A. Ashtekar, P. Singh, Loop quantum cosmology: a status report. Class. Quantum Grav. 28, 213001 (2011). doi:10.1088/ 0264-9381/28/21/213001. arXiv: 1108.0893

108. K. Banerjee, G. Calcagni, M. Martín-Benito, Introduction to loop quantum cosmology. SIGMA 8, 016 (2012). doi:10.3842/ SIGMA.2012.016. arXiv:1109.6801

109. P. Singh, Loop cosmological dynamics and dualities with Randall-Sundrum braneworlds. Phys. Rev D 73, 063508 (2006). doi:10.1103/PhysRevD.73.063508. gr-qc/0603043

110. A. Ashtekar, T. Pawłowski, P. Singh, Quantum nature of the big bang: improved dynamics. Phys. Rev. D 74, 084003 (2006). doi:10.1103/PhysRevD.74.084003. gr-qc/0607039

111. G. Calcagni, G.M. Hossain, Loop quantum cosmology and tensor perturbations in the early universe. Adv. Sci. Lett. 2, 184 (2009). doi:10.1166/asl.2009.1025. arXiv:0810.4330

112. P. Binétruy, C. Deffayet, U. Ellwanger, D. Langlois, Brane cosmological evolution in a bulk with cosmological constant. Phys. Lett. B 477, 285 (2000). doi:10.1016/S0370-2693(00)00204-5. hep-th/9910219

113. G. Calcagni, Cosmology of the Lifshitz universe. JHEP 0909, 112 (2009). doi:10.1088/1126-6708/2009/09/112. arXiv:0904.0829

114. E. Kiritsis, G. Kofinas, Hořava-Lifshitz cosmology. Nucl. Phys. B 821, 467 (2009). doi:10.1016/j.nuclphysb.2009.05.005. arXiv:0904.1334

115. S. Alexander, T. Biswas, Cosmological BCS mechanism and the big bang singularity. Phys. Rev. D 80, 023501 (2009). doi:10. 1103/PhysRevD.80.023501. arXiv:0807.4468

116. S. Alexander, T. Biswas, G. Calcagni, Cosmological BardeenCooper-Schrieffer condensate as dark energy. Phys. Rev. D 81, 043511 (2010). doi:10.1103/PhysRevD.81.043511. arXiv:0906.5161 (erratum ibid. D 81, 069902 (2010). doi:10. 1103/PhysRevD.81.069902) 